\title{
History matching of dual continuum reservoirs-preserving consistency with the fracture model
}

\author{
Pål Næverlid Sævik ${ }^{1}$ (D) Martha Lien $^{3}$ - Inga Berre ${ }^{1,2}$
}

Received: 19 August 2016 / Accepted: 26 February 2017 / Published online: 9 March 2017

(C) The Author(s) 2017. This article is published with open access at Springerlink.com

\begin{abstract}
Ensemble- and optimization-based parameter estimation is commonly used to calibrate simulation models of fractured reservoirs to measured data. Traditionally, statistical data on small-scale fractures are upscaled to a dual continuum model in a single step, and the subsequent history matching procedure makes adjustments to the upscaled parameters. In this paper, we show that the resulting reservoir models may be inconsistent with the initial fracture description, meaning that the reservoir parameters do not correspond to a physically valid combination of fracture parameters. A number of numerical examples is provided, which illustrate why and when the problem occurs. We utilize an invertible analytical fracture upscaling method, and deviations from the fracture model can thus be quantified in each case. We show that consistency with the fracture model is preserved if fracture parameters are history matched directly, if the relation between inversion variables and fracture parameters is linear, or if an unbiased Bayesian sampling method is used. We also show that preserving consistency is less important if the uncertainty of the fracture upscaling method is large.
\end{abstract}

Keywords History matching $\cdot$ Fractures $\cdot$ Upscaling $\cdot$ Dual continuum models

\footnotetext{
Pål Næverlid Sævik

pal.saevik@uib.no

1 University of Bergen, P.O. Box 7800, 5020 Bergen, Norway

2 Christian Michelsen Research, Fantoftvegen 38, 5072 Bergen, Norway

3 Octio AS, Kanalveien 119, 5068 Bergen, Norway
}

\section{Introduction}

Fractures in geological formations are of importance in petroleum production, groundwater contamination assessment, geothermal energy production, and $\mathrm{CO}_{2}$ storage. In all of these applications, assisted history matching through residual minimization or bayesian inversion is commonly applied [17]. A particular challenge with fractured reservoirs is that the reservoir parameters, such as permeability and porosity, originates from upscaling of a fracture network geometry. By perturbing the reservoir parameters individually to match production history, one runs the risk of creating parameter combinations that are inconsistent with the underlying fracture description, meaning that the perturbed parameters may correspond to improbable or impossible fracture configurations. In this paper, we investigate whether or not problems of this kind may occur during history matching of a dual-continuum reservoir.

We choose to put our emphasis on the role of fractures in petroleum engineering, where both upscaling and history matching are routinely used. Typically, fractured petroleum reservoirs are modeled using the dual-porosity model of Warren and Root [24], with the multi-phase extensions given by Kazemi et al. [10]. Fractures can have a positive, neutral, or negative effect on petroleum production, depending on the fracture network characteristics and the wettability of the reservoir [5]. Even though the presence of fractures generally enhances the permeability of the reservoir, it may also severely restrict the use of water injection, since injected water may bypass the oil residing in the porous matrix by flowing directly through the fractures. A proper characterization of the fractures and their physical properties is therefore essential in order to devise a successful production strategy. Many sources of 
fracture information may be available to the field operator, for instance, well logs [5, 12], dual-porosity well tests [7], seismic data [18], outcrop analogs, and core samples.

To ensure that the history matched reservoir stays consistent with the underlying fracture model, one may choose to history match the fracture parameters directly, and include fracture upscaling as an integral part of the parameter inversion workflow, as illustrated in Fig. 1. In the current work, we compare this method with the more traditional approach of targeting the reservoir parameters. A minor downside of using fracture parameters as inversion variables, is the added computational run time associated with the upscaling step. This disadvantage can be eliminated by using analytical upscaling methods (e.g., [13, 20, 21]), which have negligible computational cost. If numerical upscaling is required, one may first upscale a selected set of fracture parameters in a pre-processing stage and interpolate between them during history matching.

Using fracture parameters as primary inversion variables also facilitates joint inversion with other data types, such as seismic or electromagnetic data. Couplings of this type has been suggested, e.g., by Jakobsen et al. [8] and Sharani et al. [22]. In these applications, fracture parameters are the natural choice of inversion variables, since all the relevant physical properties can be calculated from them.

We begin this paper with a section on fracture upscaling, where we describe the analytical upscaling method used in our numerical examples. We employ an invertible fracture upscaling scheme, which enables us to quantify how much a given reservoir model deviates from a physically valid fracture description.

Then follows a section on history matching methods, where we outline three different techniques; the rejection method, the Ensemble Kalman Filter and the Randomized Maximum Likelihood method. We also explain why some

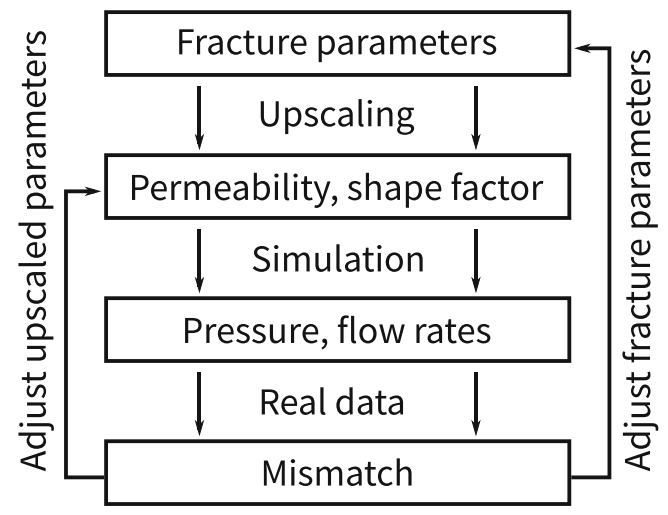

Fig. 1 Traditional and alternative history matching loop. Left: Traditional history matching loop, where upscaling is only performed initially. Right: Alternative history matching loop, where upscaling is an integral part of the procedure of these inversion methods are likely to generate fractureinconsistent reservoir models unless fracture parameters are history matched directly.

Finally, we apply the history matching methods to numerical test cases, for different choices of inversion variables. We also investigate the effect model nonlinearity, upscaling uncertainty and the prior distribution variance. Together, these examples are meant to give the reader an intuitive understanding of when fracture-inconsistent reservoir parameters may occur and what the consequences may be.

\section{Fracture upscaling model}

Upscaling of fracture data to reservoir simulator parameters can be done either through numerical or analytical upscaling. In numerical upscaling, a geometric representation of the fracture network is generated and meshed. A set of boundary conditions is applied, and the resulting flux through the matrix-fracture system is calculated $[6,9]$. In analytical upscaling, the true fracture network is represented by a simplified, idealized geometry, whose properties is calculated analytically $[13,15,21]$. The upscaled parameters of interest are the effective permeability, porosity, and the transfer coefficient (for dual-continuum models) or the pseudo relative permeability function (for singlecontinuum, multi-phase models).

In a history matching application, analytical fracture upscaling is arguably the better choice, at least when fracture parameters are used as inversion variables. This is primarily because of the computational cost, which may be large for numerical upscaling methods. Another benefit of analytical methods is that gradients can be obtained directly, which is important for gradient-based inversion methods.

In the following paragraphs, we describe the upscaling method used in our examples. We have intentionally chosen a simplified analytical model to provide a clean and intuitive illustration of the main concepts. Analytical and numerical models that account for more complicated physics and fracture geometry are available in the literature and may easily be integrated into the workflow we propose.

\subsection{Geometry}

We assume that the fractures can be represented by randomly oriented circular discs, with perfectly smooth, parallel surfaces. We also assume that the fractures are distributed evenly in space. This is the simplest fracture model that still retains enough complexity to be illustrative.

While investigating the issues presented in this paper, models with anisotropic (Fisher-distributed) fracture orientations were considered as well $[4,14]$. The results for 
these models were very similar to those for the isotropic models.

\subsection{Porosity}

Fracture porosity is simply given by

$\phi=a A$,

where $a$ is the fracture aperture, and $A$ is the fracture density measured in surface area per bulk volume. Assuming circular fractures, the fracture density is given as

$A=\sum \pi N_{R} R^{2}$,

where $N_{R}$ is the number per volume of fractures with radius $R$, and the sum is over all possible fracture radii $R$.

The above model is valid for fractures with smooth, parallel surfaces. In the case of rough-walled fractures, the aperture $a$ in Eq. 1 represents the average mechanical aperture.

\subsection{Transfer coefficient}

The transfer coefficient, which describes how freely the fluid can flow between the fracture and matrix media, is commonly calculated using an expression by Kazemi et al. [10]. The expression was originally formulated for three orthogonal, infinitely extending fracture sets,

$\sigma=4\left(\frac{1}{L_{1}^{2}}+\frac{1}{L_{2}^{2}}+\frac{1}{L_{3}^{2}}\right)$,

where $L_{1}, L_{2}$, and $L_{3}$ are fracture spacings in each orthogonal direction. Noting that fracture spacing is the inverse of fracture density, an alternative formulation can be used,

$\sigma=4\left(A_{1}^{2}+A_{2}^{2}+A_{3}^{2}\right)$

where $A_{1}, A_{2}$ and $A_{3}$ are the fracture densities of each fracture set. Assuming equal densities in all directions, we have

$\sigma=\frac{4}{3} A^{2}$

where $A$ is the total fracture density.

\subsection{Permeability}

The effective fracture permeability is calculated using the method of Mourzenko et al. [13],

$K=\frac{2}{3} f T A$,

where $K$ is the effective permeability of the fracture network, $f$ is the connectivity, $T$ the fracture transmissibility and $A$ the fracture density. The equation was originally formulated for anisotropic fracture orientations and more general fracture shapes, but this is not considered here.
The fracture transmissibility is calculated using the idealized cubic law,

$T=\frac{a^{3}}{12}$.

In the case of rough-walled fractures, the aperture $a$ in Eq. 7 is substituted by the hydraulic aperture, which may be significantly smaller than the average mechanical aperture. Models that relate the hydraulic and mechanical aperture are available in the literature, see for instance the review by Singhal and Gupta [23].

The connectivity $f$ is calculated from fracture parameters as

$f=\frac{\max (0, \beta A-2.41)^{2}}{\beta A(\beta A+3.1)}$,

$\beta=\pi^{2} R_{a v g}$,

where $R_{a v g}$ is the density-weighted average fracture radius. Equation 8 is based on experimental fitting to numerical data, we refer to Mourzenko et al. [13] for details.

\subsection{From upscaled parameters to fracture parameters}

As a tool for analyzing fracture-related correlations in history-matched reservoir models, we introduce here the inverse of the upscaling model. Given the fracture parameters $a, A$ and $R_{a v g}$, the upscaled parameters are given as

$K=\frac{1+\delta}{18} f a^{3} A$,

$\sigma=\frac{4}{3} A^{2}$

$\phi=a A$,

where the connectivity $f$ is defined by Eq. 8. In Eq. 10, we have introduced the unknown upscaling error $\delta$, which represents the inaccuracy of Eqs. 7 and 8. It may also be used to represent the uncertainty of the average fracture size $R_{\text {avg. }}$.

The mapping from $a, A, \delta$ to $K, \sigma, \phi$ is a bijective mapping unless the fracture network is fully disconnected, a case which we do not consider in this work. The inverse of Eqs. 10-12 can be calculated explicitly,

$$
\begin{aligned}
A & =\frac{1}{2} \sqrt{3 \sigma}, \\
a & =\frac{2 \phi}{\sqrt{3 \sigma}}, \\
1+\delta & =\frac{27 K \sigma}{2 f \phi^{3}} .
\end{aligned}
$$

In the above set of equations, Eq. 15 is particularly important, since $\delta$ effectively describes the deviation from the fracture upscaling model. If the value of $\delta$ computed from Eq. 15 is much larger than the upscaling uncertainty, it 
indicates that the upscaled parameters are inconsistent with the underlying fracture description.

\section{History matching methods}

History matching is the process of modifying reservoir parameters in such a way that the model agrees with observed reservoir behavior. The ultimate goal is to obtain a simulation model that is more predictive of future events. To reach this goal, the model should not only match previous observations, but also be physically realistic. If unphysical parameters are specified, the model may have poor predictive power even though it matches historical field data.

In this paper, we are concerned with keeping the reservoir parameters consistent with the underlying fracture model during history matching. By this, we mean that the permeability, porosity, and transfer coefficient should always correspond to a feasible set of fracture parameters. This is not always the case. For instance, a grid block with low porosity, a small transfer coefficient and high permeability is not consistent with the fracture model described in Section 2. As seen by Eqs. 13-14, a small porosity and transfer coefficient indicate a small fracture density and aperture, which is incompatible with having a large permeability.

As explained in the following paragraphs, inconsistent parameters may arise if upscaled reservoir parameters are used as primary variables during history matching. An alternative approach is to history match the fracture parameters directly, and include fracture upscaling in the inversion workflow (Fig. 1). This way, a fracture description of the reservoir is obtained directly, and the problem of inconsistent parameters is eliminated.

\subsection{Bayesian inversion framework}

To explain why parameters may become inconsistent with the fracture model, we employ the Bayesian framework, which is commonly used in reservoir history matching. In this framework, the model parameters $m$ are viewed as stochastic variables, each having a prior distribution representing the initial parameter uncertainty. The forward model $g$ (the reservoir simulator) is a function that takes model parameters as input, and gives data (typically well pressure, flow rate and fluid composition) as output. The data is compared with measurements $d$, and a noise-weighted mismatch is computed. As is common, we assume here that the noise is gaussian with standard deviation $\epsilon$. This gives a normally distributed likelihood with mean $d$ and standard deviation $\epsilon$. The parameters' posterior distribution is calculated as the product of the prior distribution and the likelihood,

$$
p_{\text {post }}(m)=k p_{\text {pri }}(m) p_{\text {likeli }}(g(m)),
$$

where $k$ is a normalization constant and $p_{\text {post }}, p_{\text {pri }}$, and $p_{\text {likeli }}$ are the probability densities for the posterior, prior, and likelihood, respectively.

Equation 16 can be used either with fracture parameters or upscaled reservoir parameters as primary variables. Let $m_{f}$ denote fracture parameters, and let $m_{r}$ be reservoir parameters. The traditional way of coupling upscaling with history matching is through two steps: The upscaling step

$m_{r}=U\left(m_{f}\right)$,

where $U$ is the upscaling model, and the history matching step,

$p_{\text {post }}^{r}\left(m_{r}\right)=k p_{\text {pri }}^{r}\left(m_{r}\right) p_{\text {likeli }}\left(g\left(m_{r}\right)\right)$.

Alternatively, this can be done in a single step using fracture parameters as primary variables,

$p_{\text {post }}^{f}\left(m_{f}\right)=k p_{\text {pri }}^{f}\left(m_{f}\right) p_{\text {likeli }}\left(g\left(U\left(m_{f}\right)\right)\right)$.

In Eqs. 18 and 19, the superscripts $r$ and $f$ denote probability densities for reservoir and fracture parameters, respectively. The probability density distributions are related as

$$
\begin{gathered}
p_{\text {post }}^{f}\left(m_{f}\right)=k^{\prime} p_{\text {post }}^{r}\left(U\left(m_{f}\right)\right), \\
p_{\text {pri }}^{f}\left(m_{f}\right)=k^{\prime \prime} p_{\text {pri }}^{r}\left(U\left(m_{f}\right)\right),
\end{gathered}
$$

where $k^{\prime}$ and $k^{\prime \prime}$ are normalization constants.

\subsection{Why and when do inconsistent parameters occur?}

Equations 18 and 19 are mathematically equivalent, so it may seem that either choice of inversion variables gives the same result. However, it is usually very difficult to generate an unbiased sample of the posterior distribution when the number of parameters is high. Common history matching methods therefore approximate the distribution in one way or another, and the choice of primary variables may therefore have a big impact. When using reservoir parameters as primary variables, one runs the risk of polluting the posterior distribution with parameter combinations that does not correspond to physically valid fracture parameters.

This is especially true if one or more of the fracture parameters, for instance, the upscaling uncertainty, is narrowly defined in the prior distribution. A simplified illustration of this situation is given in Fig. 2. If the upscaling uncertainty is small, or any of the other fracture parameters are narrowly defined, or there is a strong linear correlation in the fracture parameter space, this translates into a strong nonlinear correlation in the reservoir parameter space. This is no issue for history matching methods that preserve nonlinear correlations. But methods that only preserve linear correlations may run into problems, and generate posterior distributions that contain physically invalid parameter combinations. 
Fig. 2 The figure illustrates three alternative pathways to a history matched fracture model. 1 Apply history matching directly to the fracture parameters. 2 Apply an unbiased (exact) history matching algorithm in the reservoir parameter space. 3 Apply an approximate history matching algorithm that only preserves linear correlations, in the reservoir parameter space. If the upscaling uncertainty is small (or there is a strong linear correlation in the prior), method (3) may generate posterior distributions that contain physically invalid combinations of fracture parameters

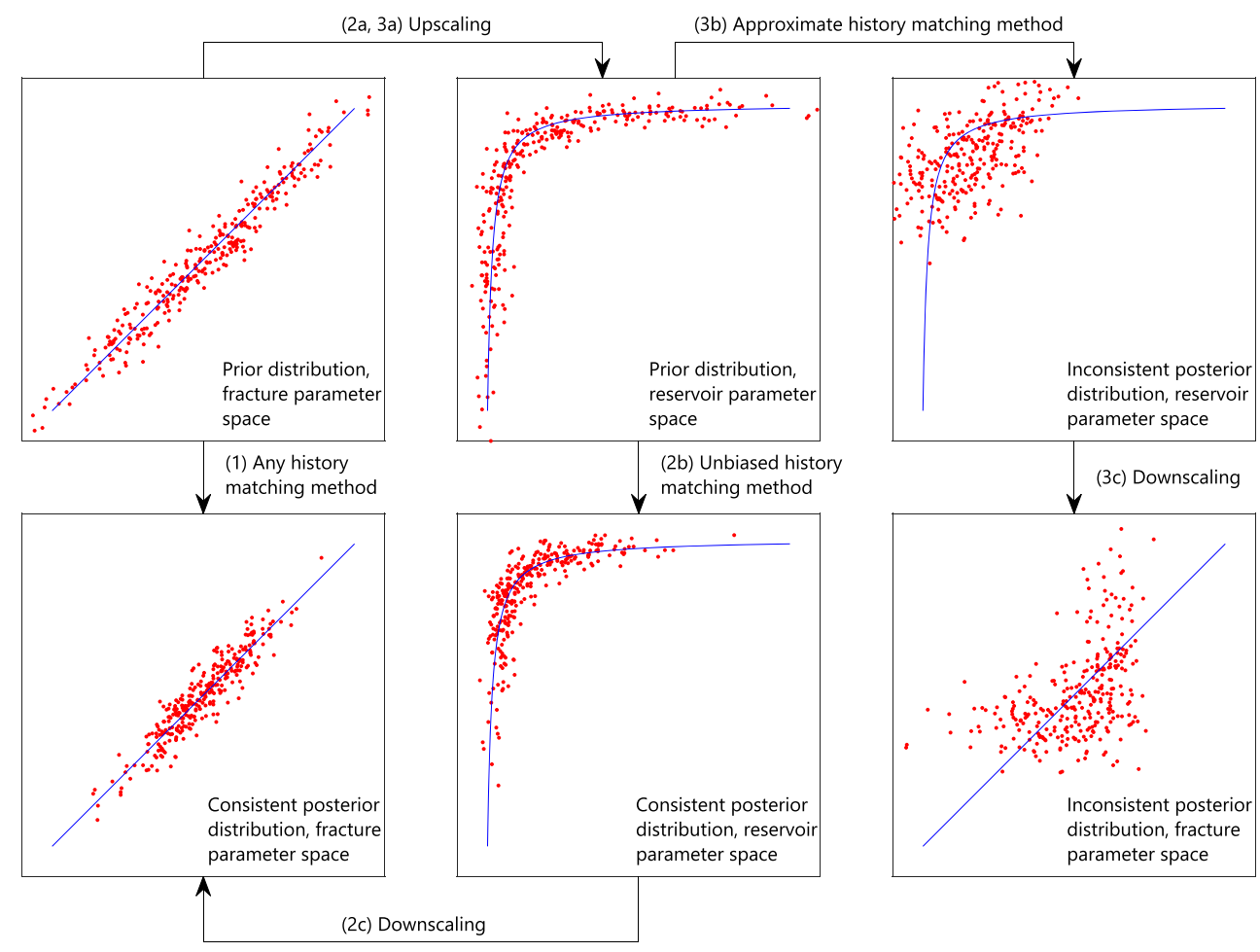

On the other hand, by using fracture parameters as primary variables, a fracture description of the reservoir is obtained directly. Thus, the problem of inconsistent parameters is avoided, regardless of the history matching method chosen.

\subsection{The rejection method}

An unbiased sample of the posterior distribution can be generated using the rejection method. For normally distributed measurement noise, the simplest version of the method is given by the following algorithm [17]: Let $m$ be a vector of parameters drawn from the prior distribution. Compute the noise-weighted squared mismatch

$z^{2}=\left\|\frac{g(m)-d}{\epsilon}\right\|^{2}$,

where $g$ is the forward model, $d$ are the measurements, and $\epsilon$ is the measurement noise. The parameters $m$ are accepted into the sample with a probability of $p=\exp \left(-0.5 z^{2}\right)$. This process continues until the desired number of samples have been generated.

Since the rejection method gives an unbiased sample, it makes no difference whether fracture parameters or upscaled parameters are used as primary variables. If upscaled parameters are used, the structure of the underlying fracture model is kept as nonlinear correlations within the prior distribution, which are preserved during inversion. However, the method becomes unfeasible when the number of parameters is high, due to the exponentially increasing number of rejected samples.

\subsection{The Ensemble Kalman Filter}

Within the last decade, the Ensemble Kalman Filter (EnKF) has gained popularity in the petroleum industry. An overview of the method and its applications in reservoir engineering is given by Aanonsen et al. [1]. The method approximates the prior by a plurigaussian distribution, calculated using an ensemble of prior reservoir models and corresponding simulation data. The model update is then feasible to compute, even when the number of parameters is high.

A simple version of the EnKF is described here. Let $M$ be a matrix representing the prior ensemble, where each column contains the parameters of one ensemble member. The model update step in the EnKF can then be expressed as $M_{\text {post }}=M+M_{\text {diff }}$, where $M_{\text {post }}$ represents the posterior ensemble, and $M_{\text {diff }}$ is given as

$M_{\text {diff }}=\Delta M \Delta D^{\top}\left[C_{D}+\Delta D \Delta D^{\top}\right]^{-1}\left(D_{o b s}-D+\epsilon\right)$.

Here, $C_{D}$ is the covariance matrix for the data noise, $D_{o b s}$ is the observed production data, and $\epsilon$ is a matrix of realized data noise. The predicted production data for the prior ensemble is given by the matrix $D$. The symbol $\Delta$ denotes the square root of the ensemble covariance in the form of

$\Delta M=(M-\bar{M}) / \sqrt{N-1}$, 
where $N$ is the number of ensemble members, and $\bar{M}$ is the ensemble mean of $M$.

In the EnKF, measurements are divided into groups, which are history matched one at a time. If all measurements are history matched simultaneously, the method is called the ensemble smoother (ES). In this case, iterative variants of Eq. 23 are recommended since the pure ES is sensitive to model nonlinearities. One simple iterative method is the multiple data assimilation (MDA) method by Emerick and Reynolds [3], where the update step is given by

$$
M_{\text {diff }}=\Delta M \Delta D^{\top}\left[C_{D}+n \Delta D \Delta D^{\top}\right]^{-1}\left(D_{o b s}-D+\epsilon\right),
$$

and $n$ is a predetermined number of iterations.

The EnKF estimate of the posterior distribution is biased towards plurigaussian distributions. If upscaled parameters are used as inversion variables, correlations between the parameters may be linearized, and the parameters may then become incompatible with the fracture model.

\subsection{Residual minimization}

A slightly different approach to history matching is residual minimization. In this approach, optimization techniques are used to modify the model parameters in order to minimize the misfit between observed measurements and data predicted from simulations. To avoid overfitting the parameters to noisy measurements, unphysical behavior (such as large oscillations) are penalized during optimization.

A particularly useful method within this class is the randomized maximum likelihood (RML) [11, 19]. In this method, optimization is performed on the objective function

$$
\begin{aligned}
f(m)= & (m-\tilde{m})^{\top} C_{M}^{-1}(m-\tilde{m}) \\
& +(g(m)-d+\epsilon)^{\top} C_{D}^{-1}(g(m)-d+\epsilon),
\end{aligned}
$$

where the first term represents the deviation from a reference reservoir, and the last term is the deviation from measurements. The vector $m$ is the model parameters, $\tilde{m}$ is the parameters of the reference model, $C_{M}$ is the prior covariance matrix, $g$ is the forward model, $d$ is the measurements, $C_{D}$ is the covariance matrix for the measurement noise, and $\epsilon$ is a vector of realized measurement noise.

In the RML, optimization of Eq. 26 is performed multiple times, each time with a different value for the realized noise $\epsilon$ and the reference model $\tilde{m}$, which is drawn from the prior distribution. This way, one obtains an assessment of the uncertainty. In fact, the method is equivalent to the Bayesian approach if the forward model $g$ is linear and the prior distribution is gaussian [16].

If reservoir parameters are used as primary variables during residual minimization, there is no guarantee that the resulting model is consistent with the underlying fracture model, since the deviation from the fracture model (given by $\delta$ in Eq. 15) is not penalized. On the other hand, if fracture parameters are history matched directly, proper penalization of $\delta$ is given by the first term of Eq. 26, and a fracture description is obtained directly.

The RML can be combined with any suitable optimization technique. In this paper, we use the LevenbergMarquardt algorithm with numerically computed gradients [2]. Since the prior covariance matrix $C_{M}$ may be singular or nearly singular, we use a truncated singular value decomposition (TSVD) to rewrite the objective function (26). Specifically, if $U \Lambda U^{\top}=C_{M}$ is a TSVD factorization, the objective function can be rewritten as

$f(x)=x^{\top} \Lambda^{-1} x+g_{x}^{\top} C_{D}^{-1} g_{x}$,

where $x=U^{\top}(m-\tilde{m})$ and $g_{x}=g(U x+\tilde{m})-d+\epsilon$.

\section{Numerical examples}

In this section, we use simple numerical examples to illustrate how history matching may give parameters that are inconsistent with the fracture model. We consider a single reservoir grid block with randomly oriented fractures of known size $R$, but unknown fracture density $A$ and aperture $a$. Table 1 gives a summary of the prior distributions used in the various examples.

In all cases, we study the effect of assimilating a noisy permeability measurement of $300 \mathrm{mD}$, for different choices of primary variables and inversion methods. The "forward model" is therefore a function that calculates the permeability from the inversion variables. If fracture parameters are used as primary inversion variables, the forward model is the permeability upscaling Eq. 10. If upscaled parameters are used, the forward model is simply a function that selects the permeability value.

\subsection{Exact, nonlinear upscaling model}

In the prior model defined by Table 1 , case 1 , the upscaling error is assumed to be zero. Inserting $\delta=0$ into Eq. 15 and solving for $f$, we obtain

$f=\frac{27 K \sigma}{2 \phi^{3}}$.

Equation 28 provides an intuitive way of testing whether the posterior reservoir parameters are consistent with the fracture model. Since the fractures in case 1 are also infinitely extending, the connectivity $f$ is equal to 1 within the prior distribution. There is no variability, so we must have $f=1$ in the posterior distribution as well, by Eq. 16. In other words, if the connectivity $f$ calculated from Eq. 28 
Table 1 Measurement data and prior distribution of fracture parameters used for the single block numerical examples

\begin{tabular}{|c|c|c|c|c|c|c|c|c|c|c|}
\hline & \multicolumn{4}{|c|}{ Prior mean $(\mu)$} & \multicolumn{4}{|c|}{ Prior scatter $(s)$} & \multicolumn{2}{|c|}{ Measurement } \\
\hline & $a$ & $A$ & $\delta$ & $R$ & $a$ & $A$ & $\delta$ & $R$ & $K_{m}$ & $\operatorname{SD}(\epsilon)$ \\
\hline Case 1 & $0.2 \mathrm{~mm}$ & $1 \mathrm{~m}^{-1}$ & 0 & $\infty$ & $0.04 \mathrm{~mm}$ & $0.4 \mathrm{~m}^{-1}$ & 0 & 0 & $300 \mathrm{mD}$ & $30 \mathrm{mD}$ \\
\hline Case 2 & $0.2 \mathrm{~mm}$ & $1 \mathrm{~m}^{-1}$ & 0 & $5 \mathrm{~m}$ & $0.04 \mathrm{~mm}$ & $0.4 \mathrm{~m}^{-1}$ & 0 & 0 & $300 \mathrm{mD}$ & $30 \mathrm{mD}$ \\
\hline Case 3 & $0.2 \mathrm{~mm}$ & $1 \mathrm{~m}^{-1}$ & 0 & $5 \mathrm{~m}$ & $0.08 \mathrm{~mm}$ & $0.8 \mathrm{~m}^{-1}$ & 0 & 0 & $300 \mathrm{mD}$ & $100 \mathrm{mD}$ \\
\hline Case 4 & $0.2 \mathrm{~mm}$ & $1 \mathrm{~m}^{-1}$ & 0 & $5 \mathrm{~m}$ & $0.04 \mathrm{~mm}$ & $0.4 \mathrm{~m}^{-1}$ & 0.1 & 0 & $300 \mathrm{mD}$ & $30 \mathrm{mD}$ \\
\hline Case 5 & $0.2 \mathrm{~mm}$ & $1 \mathrm{~m}^{-1}$ & 0 & $5 \mathrm{~m}$ & $0.08 \mathrm{~mm}$ & $0.8 \mathrm{~m}^{-1}$ & 0.1 & 0 & $300 \mathrm{mD}$ & $100 \mathrm{mD}$ \\
\hline
\end{tabular}

The fracture parameters $a, A, \delta$ and $R$ are defined in Section 2. Each parameter is uniformly distributed with range $[\mu-s, \mu+s]$. The variable $K_{m}$ is the measured permeability. The measurement noise is gaussian with standard deviation $\operatorname{SD}(\epsilon)$

is different than 1 in the posterior distribution, then the reservoir parameters are not consistent with the fracture model.

Figure 3a plots the calculated value of $f$ for 100 samples of the posterior distribution, using reservoir parameters as primary variables. The values are plotted against fracture density as defined by Eq. 13. Three different history matching methods are employed: the rejection method, the ES, and the RML.

The rejection method predicts a connectivity of $f=1$ everywhere, which is the correct result. This is not surprising, since the rejection method gives an unbiased estimate of the posterior distribution. Even though reservoir parameters are used as inversion variables, the underlying fracture model is kept as nonlinear correlations in the prior distribution. These correlations are preserved during history matching.

On the other hand, the posterior parameters obtained using the ES and RML methods clearly break with the fracture model. The methods give values of $f$ in the range between 0.5 and 1.5, and an artificial correlation between fracture density and connectivity is introduced as well. Both methods give nearly identical results, which is due to the fact that ES and RML are equivalent if the forward model is linear. Recall that the forward model simply selects the permeability value, which is clearly a linear operation.
Fig. 3 Posterior fracture connectivity as calculated by Eq. 28, plotted against fracture density as defined by Eq. 13 . The measurement data and prior distribution parameters are given by Table 1 , case 1

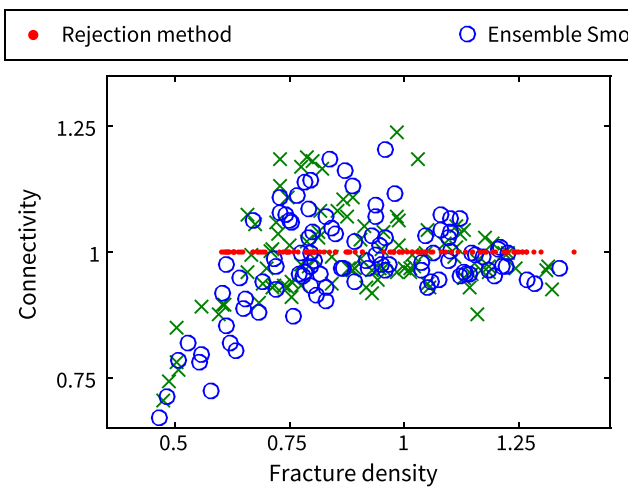

(a) Case 1. Inversion variables: Reservoir parameters

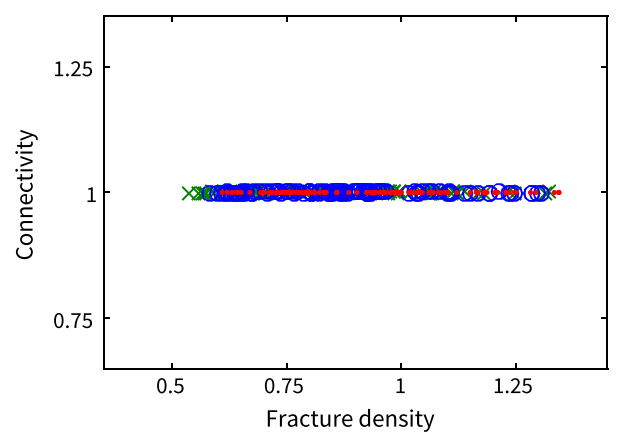

(c) Case 1. Inversion variables: Logarithm of reservoir parameters

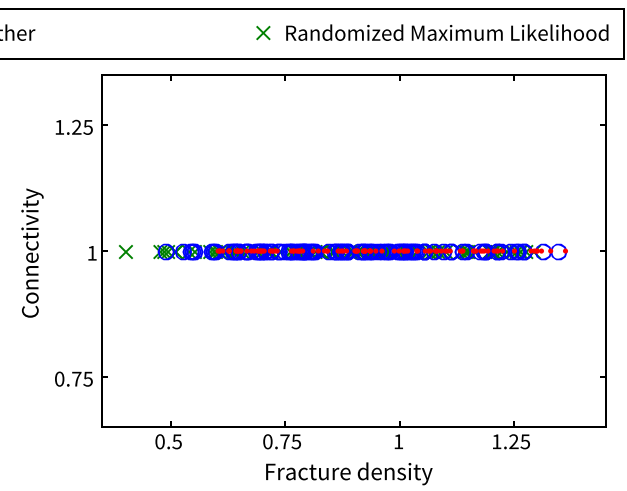

(b) Case 1. Inversion variables: Fracture parameters

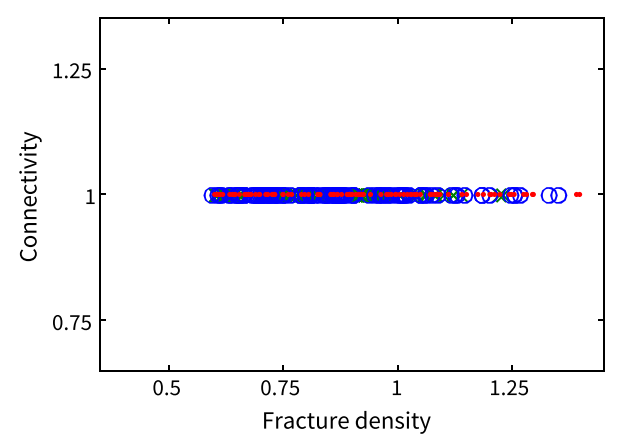

(d) Case 1. Inversion variables: Logarithm of fracture parameters 
In comparison, Fig. 3b shows the value of $f$ when fracture parameters are used as primary variables. In this case, reservoir parameters are calculated from the fracture parameters using Eqs. 10-12, which trivially leads to $f=1$ for all samples.

\subsection{Exact, linear upscaling model}

An alternative choice of inversion variables is the logarithm of the upscaled parameters, which is often used to ensure that the reservoir parameters stay positive. Figure $3 \mathrm{c}$ shows the calculated value of $f$ in this case. Figure $3 d$ shows the corresponding results when the logarithm of the fracture parameters are used as inversion variables.

Interestingly, the ES and RML now correctly predicts $f=1$ for either choice of inversion variables. To see why this happens, note that both methods are invariant under a linear change of variables, which can be verified by inspecting Eqs. 23 and 26, respectively. Furthermore, the change from fracture parameters to reservoir parameters is linear if the logarithm transform is used, which is verified by taking the logarithm of Eqs. 10-12.

If the logarithm of reservoir parameters are used as inversion variables, the structure of the fracture model is retained as linear correlations within the prior distribution, which is preserved by the ES and RML during inversion. The posterior distribution is therefore fully consistent with the original fracture model.

\subsection{Exact upscaling model with finite fractures}

Many fracture systems cannot be modeled as fully connected. In case 2, fractures are assumed to have a radius of $5 \mathrm{~m}$, in which case connectivity is a function of fracture density as described by Eq. 8 . The relation between fracture parameters and reservoir parameters is therefore nonlinear even if the logarithm transform is used.

Figure $4 \mathrm{a}$ shows the posterior connectivity when the inversion variables are the pure reservoir parameters, with no logarithm transform. The rejection method predicts a strict, weakly nonlinear relationship between fracture density and connectivity in the posterior distribution, which is consistent with the original connectivity model (8). The ES and RML methods significantly overestimate the connectivity, and there is a high variability within the results.

In comparison, Fig. 4b shows the posterior connectivity when the logarithm transform is used. The ES and RML methods predict a strong linear correlation in this case, which is very close to the true result. The example shows that the logarithm of reservoir parameters may be an adequate choice of inversion variables, even for a realistic nonlinear density/connectivity model.

The picture changes slightly if we have a larger uncertainty in the model. In case 3 , the prior distribution include a few models where the fracture density is close to the percolation threshold, and the uncertainty of the permeability measurement is large. This magnitude of uncertainty may
Fig. 4 Posterior fracture connectivity as calculated by Eq. 28, plotted against fracture density as defined by Eq. 13 . The measurement data and prior distribution parameters are given by Table 1

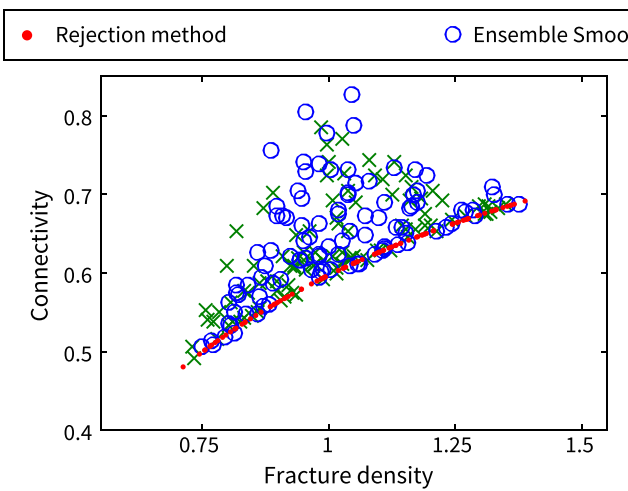

(a) Case 2. Inversion variables: Reservoir parameters.

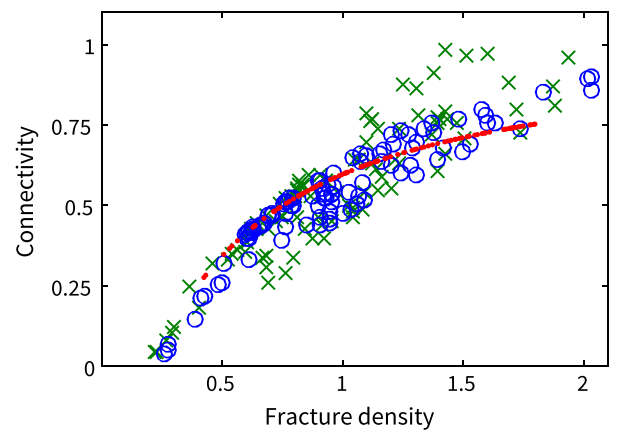

(c) Case 3. Inversion variables: Logarithm of reservoir parameters.

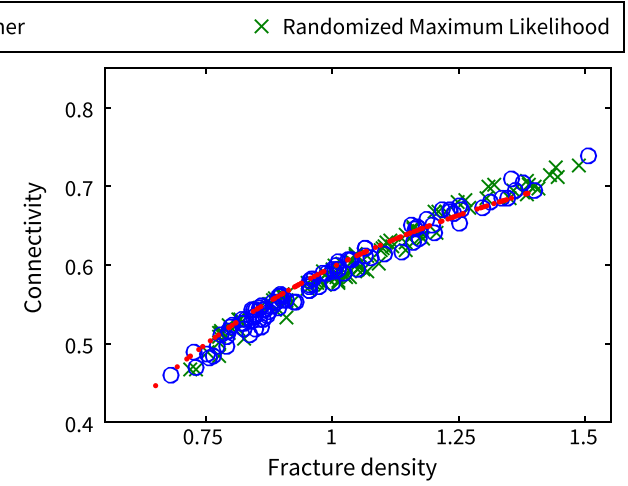

(b) Case 2. Inversion variables: Logarithm of reservoir parameters

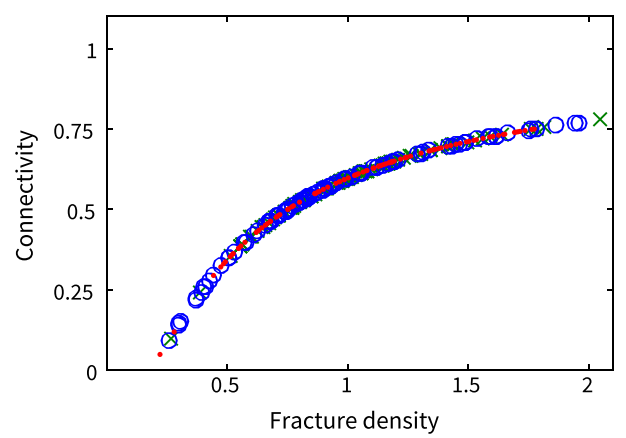

(d) Case 3. Inversion variables: Logarithm of fracture parameters. 
resemble a grid block conditioned to a hydraulic well test some distance away.

Since the prior distribution might contain zero permeability values below the percolation threshold, we can not use the logarithm transform directly. Instead, we use the variable $\log (K+1)$, where the fracture permeability $K$ is measured in millidarcy. For the porosity $\phi$ and transfer coefficient $\sigma$, we still use the logarithm directly.

Figure $4 \mathrm{c}$ shows samples from the resulting posterior distribution. The deviation from the rejection method is larger in this case, indicating a significant departure from the original fracture model. The example shows that the nonlinearity of the upscaling step may be more dominating if the prior uncertainty and data noise is high.

In contrast, by using fracture parameters as inversion variables, consistency with the fracture model is preserved regardless of the inversion method and the nonlinearity of the upscaling model. The resulting posterior connectivity distribution is shown in Fig. 4d.

\subsection{Uncertain upscaling models}

In the presence of upscaling errors, weak deviations from the fracture model may be interpreted as imperfections in the model. Cases 4 and 5 are similar to cases 2 and 3, except that an upscaling error of $10 \%$ has been added. This implies that there is a strong, but not perfect nonlinear correlation between fracture density and connectivity.
Figure 5 shows some of the results for cases 4 and 5. The rejection method correctly predicts a scattered distribution within a restricted area, reflecting that the relation between fracture density and connectivity is uncertain. In Fig. 5a, the relation between fracture parameters and reservoir parameters is highly nonlinear, since the logarithm transform is not used. The posterior distributions obtained from the ES and RML methods in this case deviate strongly from the original connectivity model (8), and extend beyond the range predicted by the rejection method. Thus, the deviations are too large to be interpreted as upscaling uncertainties.

In Fig. 5b, the logarithm of the reservoir parameters are used as inversion variables. The nonlinearity is greatly reduced, and the upscaling error dominates the deviations from the connectivity model.

Posterior connectivity results for case 5 are shown in Figure 5c, d, where the inversion variables are logarithmic reservoir and logarithmic fracture parameters, respectively. Compared with case 4 , the prior distribution is broader and the measurement uncertainty is larger, which leads to a more nonlinear relationship between fracture and reservoir parameters. In Fig. 5c, the connectivity obtained from the ES and RML deviate significantly from the reference solution provided by the rejection method. This indicates that the deviations cannot be interpreted as imperfections in the upscaling model.

Consistency with the fracture model can always be preserved by using fracture parameters as inversion variables.
Fig. 5 Posterior fracture connectivity as calculated by Eq. 28, plotted against fracture density as defined by Eq. 13 . The measurement data and prior distribution parameters are given by Table 1

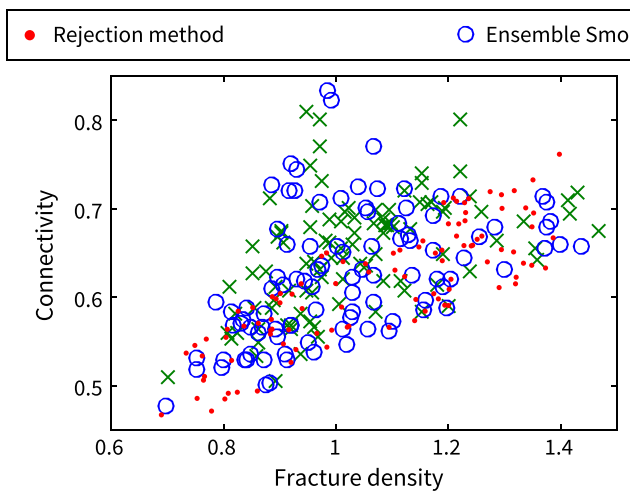

(a) Case 4. Inversion variables: Reservoir parameters.

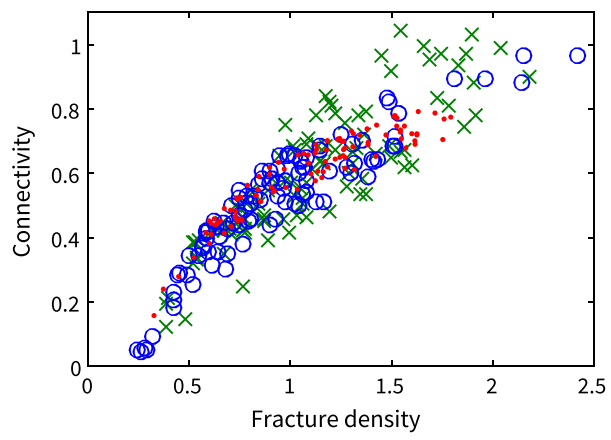

(c) Case 5. Inversion variables: Logarithm of reservoir parameters.

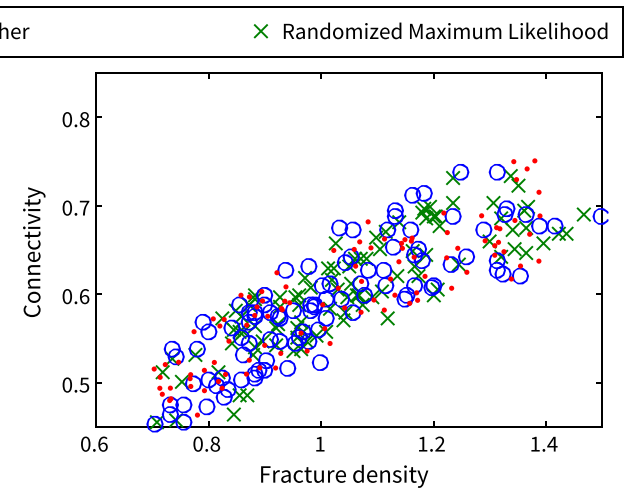

(b) Case 4. Inversion variables: Logarithm of reservoir parameters.

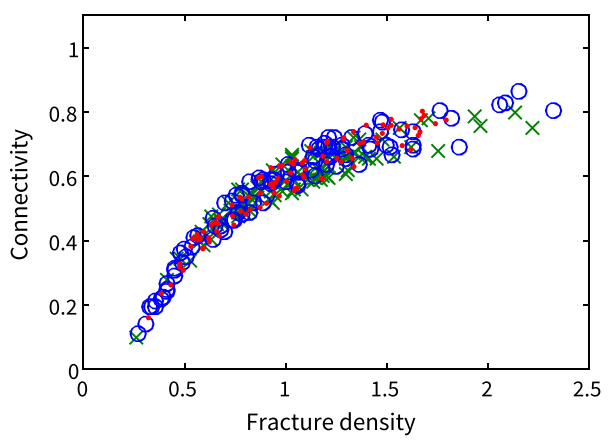

(d) Case 5. Inversion variables: Logarithm of fracture parameters. 
Fig. 6 The apparent upscaling error in the posterior distribution, as calculated by Eq. 15. Each histogram summarizes the value of $\delta$ for 10 000 posterior samples. The area under each curve is equal to 1 .

The measurement data and prior distribution parameters are given by Table 1

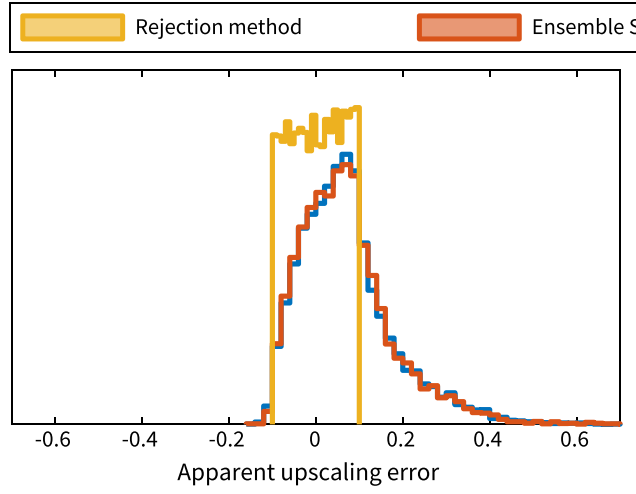

(a) Case 4. Inversion variables: Reservoir parameters.

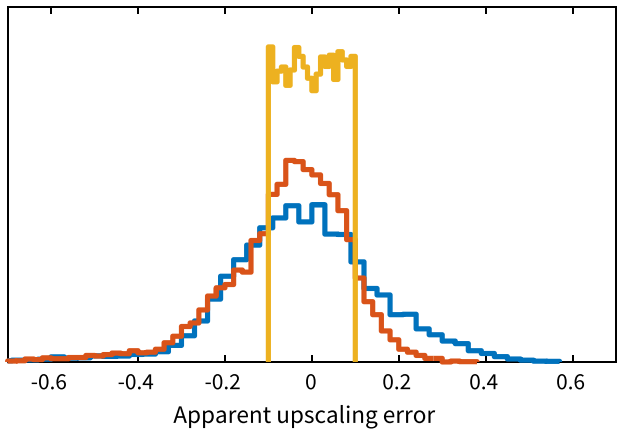

(c) Case 5. Inversion variables: Logarithm of reservoir parameters.
Randomized Maximum Likelihood

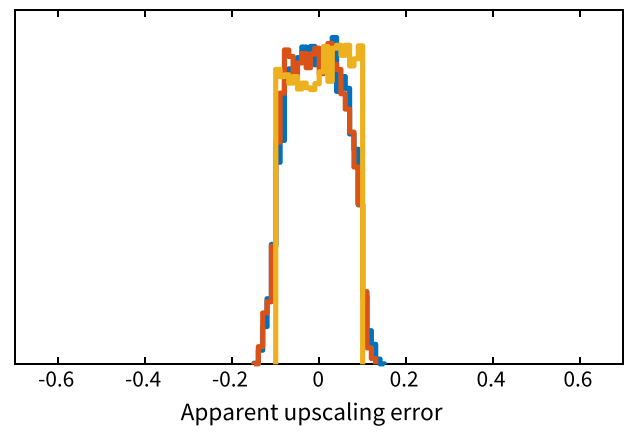

(b) Case 4. Inversion variables: Logarithm of reservoir parameters.

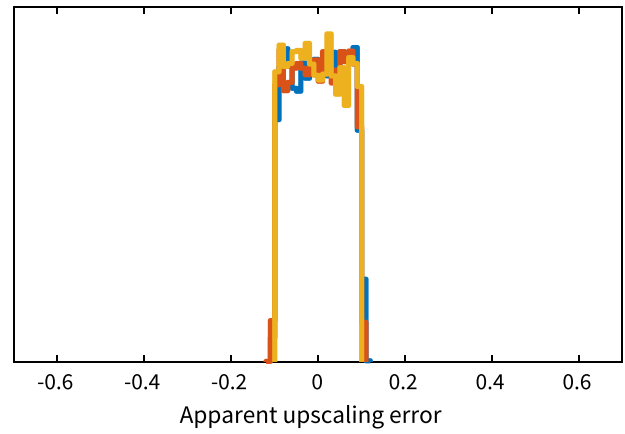

(d) Case 5. Inversion variables: Logarithm of fracture parameters.
This is demonstrated in Fig. 5d, where the permeability measurement of case 5 is assimilated by history matching the fracture parameters directly. The resulting distribution agrees with the rejection method and is consistent with a $10 \%$ spread around the connectivity model (8).

Another way of visualizing the results from cases 4 to 5 is provided by Fig. 6 . The figure presents histograms for the apparent upscaling error $\delta$, as given by Eq. 15, which measures the deviation from the fracture model in the posterior distribution. Each histogram summarizes the value of $\delta$ for 10000 posterior samples. The histograms are normalized by their area, i.e., the area under each curve is equal to 1 .

Figure 6 shows the same patterns as Fig. 5. The rejection method always give parameter combinations that deviate less than $10 \%$ from the connectivity model, as it should. For

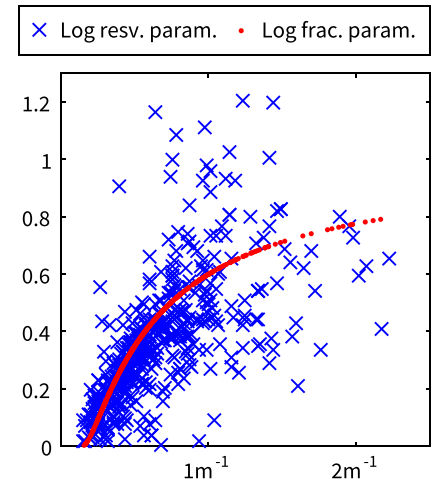

(a) Fracture connectivity vs. density, for 500 random grid blocks in the posterior ensemble. Legend indicates the choice of inversion variables.

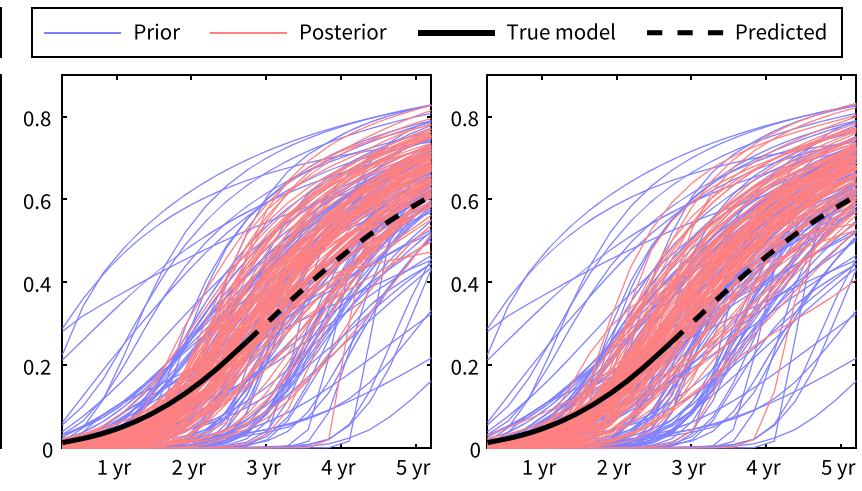

(b) Prior and posterior water cut data, using (c) Prior and posterior water cut data, using logarithm of fracture parameters as inversion logarithm of reservoir parameters as inversion variables.
Fig. 7 Results from the two-dimensional test case described in Table 2. The inversion method is multiple data assimilation, Eq. 25, with four iterations. An ensemble of 100 samples is used, one of the prior samples is used as the true model. Similar results are obtained when using other samples as the true model. The assimilated data are the volume production rate, injection pressure, and water cut, measured at 10 equally spaced intervals. The measurement noise for the three data types is gaussian with standard deviations 10 bar, $5 \%$ and 1 $\mathrm{m}^{3} /$ day, respectively 
the ES and RML methods, the results depend on the choice of inversion variables. In Fig. 6a, c, where the upscaling model is highly nonlinear, the deviation from the fracture model is larger than the upscaling uncertainty for a significant portion of the posterior samples.

In contrast, the upscaling model in Fig. $6 \mathrm{~b}$ is only weakly nonlinear, and the results for the RML and ES methods are in close harmony with the rejection method. Finally, Fig. $6 \mathrm{~d}$ shows the results for case 5 when the logarithm of fracture parameters are used as inversion variables. In this case, a fracture description of the reservoir is obtained directly, and the value of $\delta$ does not exceed the limit of $10 \%$ defined by the prior distribution.

\subsection{A simple 2D example with prediction}

The ultimate goal of history matching is to improve the predictive power of the reservoir model. In general, a model that contains geologically unrealistic features may have suboptimal predictive capabilities, even if it matches production history. It is therefore natural to assume that history matched reservoir models which are also consistent with the underlying fracture model, may have an advantage when predicting future events.

Being consistent with the fracture model may not be important in all applications, however. The consequences of predicting from a fracture-inconsistent model depend on the data to be predicted, and their sensitivity to model parameters. An example is shown in Fig. 7, where a synthetic

Table 2 Simulation data for the quarter five spot test case

\begin{tabular}{ll}
\hline Physical dimensions & $200 \mathrm{~m} \times 200 \mathrm{~m} \times 10 \mathrm{~m}$ \\
Grid resolution & $10 \times 10 \times 1$ \\
Injection rate & $50 \mathrm{~m}^{3} /$ day \\
Prod. well pressure & $200 \mathrm{bar}^{-4}$ \\
Fluid and rock compr. & $10^{-4}$ bar $^{-1}$ \\
Relative permeability $^{\mathrm{a}}$ & $k_{r w}=S, k_{r o}=1-S$ \\
Matrix cap. pressure $^{\mathrm{a}}$ & $\frac{1-S}{1+S} \times 0.5 \mathrm{bar}$ \\
Matrix porosity $^{\text {Matrix permeability }}$ & 0.3 \\
Logarithm of fracture $^{\mathrm{b}}$ & $1 \mathrm{mD}$ \\
aperture, in mm & $\mu=\log 0.2, \sigma=\log 2$ \\
Logarithm of fracture & \\
density, in $\mathrm{m}^{-1 \mathrm{~b}}$ & $\mu=\log 0.5, \sigma=\log 2$ \\
Spatial correlation of & \\
fracture parameters & Gaussian, correlation \\
\hline
\end{tabular}

The model contains one rate-controlled water injection well and one pressure-controlled production well, placed at opposing corners. Fracture capillary pressure is zero

${ }^{\text {a}}$ Water saturation: $S$

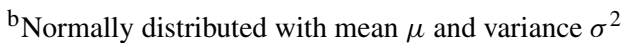

(quarter five spot), water-wet oil reservoir is history matched against water cut, bottom hole pressure and liquid production rate. The model specifications are given in Table 2. From Fig. 7a, we see that the posterior ensemble is not consistent with the fracture upscaling model unless fracture parameters are history matched directly. Nevertheless, Fig. 7b, c shows that the predicted future water cut is very similar for both choices of inversion variables.

Part of the reason is that the water cut has very little sensitivity to fracture porosity, since most of the injected water migrates to the water-wet rock matrix. Thus, fracture porosity largely becomes a redundant parameter, and the reservoir model is predictive even though fracture porosity is not correctly estimated.

\section{Summary and discussion}

In this paper, we have shown that common history matching methods may generate reservoir models that are inconsistent with the underlying fracture model, meaning that the reservoir parameters (permeability, porosity and transfer coefficient) does not correspond to a physically valid combination of fracture parameters (aperture, density and fracture size).

We have demonstrated that significant deviations from the fracture model can occur for the ensemble smoother (ES) and the randomized maximum likelihood (RML) methods, if the relation between the inversion variables and the fracture parameters is nonlinear. It is expected that similar deviations would occur with other ensemble- or optimization-based history matching methods.

The problem can easily be avoided by using fracture parameters as inversion variables. With this choice, a fracture-oriented description of the reservoir is obtained directly. The approach requires fracture upscaling to be included in the history matching loop, but the added computational cost is negligible if analytical upscaling is used.

To quantify deviations from the fracture model, we employed an invertible analytical upscaling scheme, which enabled us to convert reservoir parameters to the closest matching set of fracture parameters. Interestingly, deviations from the fracture model were much smaller if the logarithm of the reservoir parameters were used as inversion variables, compared with using the reservoir parameters directly. The reason is that the logarithm transform strongly reduced the nonlinearity of the fracture upscaling function. For fully connected fracture networks, logarithmic reservoir parameters gave just as good results as using fracture parameters as inversion variables.

Our numerical examples also demonstrated that moderate deviations from the fracture model may be acceptable if the upscaling method is uncertain. In this case, deviations from 
the fracture model may be interpreted as inaccuracies in the upscaling method.

The importance of having a reservoir description that is consistent with the fracture model depends on the specific application. Our numerical examples include a simple history matching case with a quarter five spot reservoir model, where consistency with the fracture model does not seem to give a clear predictive advantage.

Consistency with the fracture model is likely to be more important if the assimilated and predicted data are of different types. Examples include the prediction of water cut from a dual porosity well test or the prediction of heat transfer rate from a tracer test in a geothermal reservoir. In these applications, the data to be predicted are indirectly coupled to the assimilated data through the fracture model. Thus, keeping the parameters consistent with a fracture description of the reservoir could potentially improve the predictive power of the model. The same argument applies to joint inversion applications, such as joint inversion of seismic and electromagnetic data, where fracture parameters may be a natural common set of parameters linking the two data types. An investigation of these issues is, however, beyond the scope of this paper.

In cases where consistency with the fracture model is important, fracture parameters should be used as inversion variables. This choice allows one to directly constrain and correlate the fracture density, aperture, and connectivity through a prior distribution or a penalty term, in accordance with prior geological knowledge. If upscaled reservoir parameters are used as inversion variables, the inversion methods may generate history matched models that does not correspond to physically valid fracture parameters.

Acknowledgments The first author gratefully acknowledges the financial support of Statoil ASA through the Akademia agreement and The Norwegian Academy of Science and Letters through the VISTA program.

Open Access This article is distributed under the terms of the Creative Commons Attribution 4.0 International License (http://creativecommons. org/licenses/by/4.0/), which permits unrestricted use, distribution, and reproduction in any medium, provided you give appropriate credit to the original author(s) and the source, provide a link to the Creative Commons license, and indicate if changes were made.

\section{References}

1. Aanonsen, S.I., Nævdal, G., Oliver, D.S., Reynolds, A.C., Vallés, B.: The ensemble Kalman filter in reservoir engineering-a review. SPE J. 14(03), 393-412 (2009). doi:10.2118/117274-PA

2. Aster, R.C., Borchers, B., Thurber, C.H.: Parameter estimation and inverse problems, 1 edn. Elsevier Inc (2005)
3. Emerick, A.A., Reynolds, A.C.: Ensemble smoother with multiple data assimilation. Comput. Geosci. 55, 3-15 (2013). doi:10.1016/j.cageo.2012.03.011

4. Fisher, R.: Dispersion on a sphere. Proc R Soc A: Math Phys Eng Sci 217(1130), 295-305 (1953). doi:10.1098/rspa.1953. 0064

5. Golf-Racht, T.D.V.: Fundamentals of Fractured Reservoir Engineering. Elsevier Publishing Company (1982)

6. Gong, B., Karimi-Fard, M., Durlofsky, L.J.: Upscaling discrete fracture characterizations to dual-porosity, dual-permeability models for efficient simulation of flow with strong gravitational effects. SPE J. 13(1), 58-67 (2008). doi:10.2118/ 102491-PA

7. Horne, R.N.: Modern Well Test Analysis: A Computer-Aided Approach. Petroway, Inc., Palo Alto (1995)

8. Jakobsen, M., Skjervheim, J.A., Aanonsen, S.I.: Characterization of fractured reservoirs by effective medium modelling and joint inversion of seismic and production data. J. Seism. Explor. 16, 175-197 (2007)

9. Karimi-Fard, M., Gong, B., Durlofsky, L.J.: Generation of coarse-scale continuum flow models from detailed fracture characterizations. Water Resour. Res. 42(10), W10423 (2006). doi:10.1029/2006WR005015

10. Kazemi, H., Merrill, L., Porterfield, K., Zeman, P.: Numerical simulation of water-oil flow in naturally fractured reservoirs. Soc. Pet. Eng. J. 16(6), 317-326 (1976). doi:10.2118/5719-PA

11. Kitanidis, P.K.: Quasi-linear geostatistical theory for inversing. Water Resour. Res. 31(10), 2411-2419 (1995). doi:10.1029/95WR01 945

12. Luthi, S.M., Souhaité, P.: Fracture apertures from electrical borehole scans. Geophysics 55(7), 821-833 (1990). doi:10.1190/1.144 2896

13. Mourzenko, V.V., Thovert, J.F., Adler, P.M.: Permeability of isotropic and anisotropic fracture networks, from the percolation threshold to very large densities. Phys. Rev. E 84(3), 036307 (2011). doi:10.1103/PhysRevE.84.036307

14. Mourzenko, V.V., Thovert, J.F., Adler, P.M.: Trace analysis for fracture networks with anisotropic orientations and heterogeneous distributions. Phys. Rev. E 83(3), 031104 (2011). doi:10.1103/PhysRevE.83.031104

15. Oda, M.: Permeability tensor for discontinuous rock masses. Géotechnique 35(4), 483-495 (1985). doi:10.1680/geot.1985.35. 4.483

16. Oliver, D.S.: Minimization for conditional simulation: relationship to optimal transport. J. Comput. Phys. 265, 1-15 (2014). doi:10.1016/j.jcp.2014.01.048

17. Oliver, D.S., Reynolds, A.C., Liu, N.: Inverse Theory for Petroleum Reservoir Characterization and History Matching. Cambridge University Press, Cambridge (2008). doi:10.1017/CB O9780511535642

18. Rủger, A., Tsvankin, I.: Using AVO for fracture detection: analytic basis and practical solutions. Lead. Edge 16(10), 1429-1434 (1997). doi:10.1190/1.1437466

19. Oliver, D.S., He, N., Reynolds, A.C.: Conditioning permeability fields to pressure data (1996). doi:10.3997/2214-4609. 201406884

20. Sævik, P.N., Berre, I., Jakobsen, M., Lien, M.: A 3D computational study of effective medium methods applied to fractured media. Transp. Porous Media 100(1), 115-142 (2013). doi:10. 1007/s11242-013-0208-0

21. Sævik, P.N., Jakobsen, M., Lien, M., Berre, I.: Anisotropic effective conductivity in fractured rocks by explicit effective 
medium methods. Geophys. Prospect. 62(6), 1297-1314 (2014). doi:10.1111/1365-2478.12173

22. Shahraini, A., Ali, A., Jakobsen, M.: Characterization of fractured reservoirs using a consistent stiffness-permeability model: focus on the effects of fracture aperture. Geophys. Prospect. 59(3), 492505 (2011). doi:10.1111/j.1365-2478.2010.00934.x
23. Singhal, B.B.S., Gupta, R.P.: Applied Hydrogeology of Fractured Rocks, 2nd edn. Springer, Netherlands (2010). doi:10.1007/97890-481-8799-7

24. Warren, J., Root, P.: The Behavior of Naturally Fractured Reservoirs. Soc. Pet. Eng. J. 3(3), 245-255 (1963). doi:10.2118/ 426-PA 